NASA Contractor Report 4296

\title{
Multilevel Decomposition Approach to the Preliminary Sizing of a Transport Aircraft Wing
}

Gregory A. Wrenn and Augustine R. Dovi

CONTRACT NAS1-19000

MAY 1990 
NASA Contractor Report 4296

\section{Multilevel Decomposition Approach to the Preliminary Sizing of a Transport Aircraft Wing}

Gregory A. Wrenn and Augustine R. Dovi

Lockheed Engineering \& Sciences Company

Hampton, Virginia

Prepared for

Langley Research Center

under Contract NAS1-19000

\section{NMSA}

National Aeronautics and

Space Administration

Office of Management

Scientific and Technical Information Division 
CONTENTS

$\begin{array}{lr}\text { List of Tables } & \text { Page } \\ \text { List of Figures } & \text { iv } \\ \text { Nomenclature } & \text { v } \\ \text { Definitions } & \text { vii } \\ \text { Summary } & 1 \\ \text { Introduction } & 1 \\ \text { Decomposition Into Three Levels } & 3 \\ \text { Description of Level I } & 4 \\ \text { Description of Level II } & 6 \\ \text { Description of Level III } & 10 \\ \text { Finite Element Model } & 13 \\ \text { Results and Discussion } & 13 \\ \text { Conclusions } & 15 \\ \text { References } & 16\end{array}$




\section{LIST OF TABLES}

\section{Page}

Table 1: Constant Parameters for the Lockheed

Table 2: $\quad$ Initial and Final Optimization Parameters

Table 3: $\quad$ Computer Resource Chart for a Typical 3 Level Cycle 


\section{LIST OF FIGURES}

Page

Figure 1. Block Diagram of Multilevel Scheme 21

Figure 2. Wing Skin Distribution 21

Figure 3. Stiffened Panel Design Variables 21

Figure 4. Wing Cover Panels 21

Figure 5. Skin Thickness Distribution 22

With Realistic Initial Conditions

Figure 6. Skin Thickness Distribution 22

With Unrealistic Initial Conditions

Figure 7. Block Fuel Convergence 22

Figure 8. Block Fuel Convergence 22

Figure 9. Wing Weight Convergence 22

Figure 10. Wing Weight Convergence 22

Figure 11. Thickness/Chord Convergence 23

Figure 12. Thickness/Chord Convergence 23 


\section{NOMENCLATURE}

[A\} vector of wing panel areas

$A_{\text {flanges }} \quad$ cross sectional area of upper and lower stiffener flanges

$A_{\text {stif }}$ cross sectional area of one stiffener

$\mathrm{C}_{0-4}$ coefficients of polynomial defining wing skin distribution

DKS first derivative of the K-S function

$f$ factor relating wing skin weight to total wing structural weight.

g gravity acceleration

$g_{j k}$ bottom level behavior response equations

$\mathrm{g} \Omega$ top level constraint on structural load carrying capacity

$h_{\text {web }}$ height of stiffener web

K-S Kreisselmeier-Steinhauser function

$t_{\text {inboard }}$ equation for skin thickness of inboard portion of wing equation for skin thickness of outboard portion of wing $t_{\text {skin }}$ skin thickness of a wing panel

$t_{\text {stif }}$ smeared thickness of stiffeners on a wing panel $t_{\text {web }}$ thickness of stiffener web vector of wing panel skin thicknesses

t/c wing thickness to chord ratio

$\mathrm{W}_{\mathrm{s}}$ wing structural weight $W_{\text {skin }}$ wing skin weight non-dimensional wing semi-span

$\Delta \quad$ small change in a parameter

$\rho$

K-S factor

$\rho_{d}$

weight density of skin material 
$\begin{array}{ll}\sigma_{m} & \text { stress for load case } m \\ \Omega & \text { middle level objective function } \\ \omega_{i} & \text { bottom level objective function for the } i^{\text {th }} \text { wing panel }\end{array}$

\section{DEFINITIONS}

Behavior Sensitivity Derivatives ${ }^{1,2}$ - derivatives of system behavior response with respect to a system design variable.

Optimum Sensitivity Derivatives 3,4 - derivatives of the optimum objective function and design variables with respect to a system parameter - constant in the optimization.

Cumulative Constraint ${ }^{5}$ - a single constraint function which represents several constraints. 


\section{SUMMARY}

This report describes a multilevel/multidisciplinary optimization scheme for sizing an aircraft wing structure. A methodology using nonlinear programming in application to a very large engineering problem is presented. This capability is due to the decomposition approach. Over 1300 design variables are considered for this nonlinear optimization task. In addition, a mathematical link is established coupling the detail of structural sizing to the overall system performance objective, such as fuel consumption. The scheme is implemented as a three level system analyzing aircraft mission performance at the top level, the total aircraft structure at the middle level, and individual stiffened wing skin cover panels at the bottom level. Numerical results show effectiveness of the method and its good convergence characteristics.

\section{INTRODUCTION}

The preliminary design of a large subsonic transport aircraft is an extremely complex task. Many engineering disciplines must interact with each other to determine the final configuration. Traditionally, parametric studies involving trade-off comparisons are usually performed to meet the design objectives. These studies may include suboptimizations that perturb a known baseline configuration ${ }^{6}$ to yield the best design at the sublevels. The net result is a thumbprint sketch made from a collection of independent studies from which the "best" design may be chosen. This approach may produce a design that is not globally optimum, but instead may represent a design that is weighted toward one of the subproblems. In addition, statistical data collected from existing aircraft configurations is required as a starting point and the new design is limited to small perturbations from that baseline case ${ }^{7}$. 
An alternative method called the multilevel optimization by linear decomposition (MOLD) for optimization of large and complex engineering systems for maximum performance was formulated initially for generic engineering systems ${ }^{8,9}$ and subsequently adapted to aerospace applications ${ }^{10}$. The method has also been validated for structural design 11,12 . This report describes the implementation of MOLD for preliminary design of a large transport aircraft for the purposes of testing the method's effectiveness and convergence characteristics. Consistent with this purpose and with the resources that were available, the testing was limited to the wing as the only variable part of the aircraft, it excluded the analyses of flutter and gust response, and used constant aerodynamic wing loading.

The method is based on the use of derivatives to form approximations of the behavior of subsystems with respect to aircraft configuration design variables. This approach provides a synergistic effect by allowing the influence of the various subsystems to take part in the total design in an optimum manner. The scheme is based on the ability to linearly decompose the design problem into multiple subproblems. The top level contains the aircraft performance analysis, the middle level the total wing box structural analysis, and the bottom level the individual wing skin cover panels. Analysis and optimization are performed at each level and design information is passed between the levels in a hierarchical manner. The information passed between the levels are typically analysis results in the form of parameters and sensitivity derivatives ${ }^{3,4}$. Using this procedure, more rigorous analytical tools ${ }^{13,14}$ can be used instead of statistical estimates for the analysis of the aircraft structural components and allows the designer the freedom to investigate configurations for which there is no existing similar aircraft from which to obtain baseline data. 
Included in this report is a description of a three level system which performs a preliminary design based on performance and structural requirements. The system is applied to a large transport aircraft wing configuration to minimize block fuel by varying the structural weight and the thickness to chord ratio of the wing. The numerical results are discussed along with system convergence behavior and typical computer resource requirements.

\section{DECOMPOSITION INTO THREE LEVELS}

In this report, the wing sizing problem includes aircraft performance as well as structures disciplines. The wing design is decomposed into three separate optimization subproblems, as shown in Figure 1. The top level performs an initial analysis to provide information for the lower levels. At this time, the initial values of wing weight and thickness to chord ratio are set. Execution then continues at the middle level which uses the top level design variables as constant parameters. The middle level performs an initialization of the finite element model including the initial wing skin thickness distribution. A static analysis is performed and stresses are computed for each of the wing skin cover panels. The flow of execution progresses to the bottom level where the wing skin panels are optimized one at a time for the minimum of a cumulative constraint ${ }^{5}$ representing ștress and buckling violations. Once processing at the bottom level is completed, a single value of the cumulative constraint is passed back to the middle level along with its derivatives with respect to the constant parameters, obtained by optimum sensitivity analysis techniques ${ }^{3}$. A firstorder Taylor series is formed from these data to linearly approximate the behavior of the bottom level optimum solution. This approximation may then be used in the middle level's optimization to represent the effect the middle level skin distribution will have on panel failure without having to repeat the optimizations of each bottom level panel 
during the design process. Once an optimum solution is obtained at the middle level, sensitivity derivatives are computed with respect to the top level design variables. The top level then performs an aircraft mission and performance optimization which includes a linear approximation of the behavior of the aircraft structure as one of its constraints. The above description represents one complete cycle of the three level optimization procedure. The entire process is repeated until convergence at all levels is obtained. In the following sections, each level is discussed in more detail.

\section{DESCRIPTION OF LEVEL I}

The top level of the multilevel system uses the Flight Optimization System (FLOPS) ${ }^{15}$ to analyze the mission and performance characteristics of the aircraft configuration. FLOPS is a collection of computer programs consisting of four primary modules: (1) weights, (2) aerodynamics, (3) mission performance, (4) takeoff and landing. The weights module uses statistical/empirical equations to predict the weight of each item. Aerodynamic drag polars are generated using the Empirical Drag Estimation Technique (EDET) ${ }^{16}$ in the aerodynamics module. The mission performance module uses weights, aerodynamic data, and an engine deck to calculate performance. Based on energy considerations, an optimum climb profile is flown to the start of the cruise condition. The cruise segment is flown at an optimum altitude for maximum range or at optimum Mach number for maximum endurance. Takeoff and landing analysis includes the evaluation of constraints on approach speed, missed approach climb gradient, second segment climb gradient, landing field length, and takeoff field length. FLOPS can be used to optimize aircraft parameters such as $t / c$, aspect ratio, and sweep angle in order to minimize gross weight or block fuel, or to maximize range using the Fiacco and McCormick interior penalty function coupled with the Davidon-Fletcher-Powell method 17 . Modifications were made to 
FLOPS to include wing skin structural weight as a design variable and to use the Relational Information Management System (RIM) ${ }^{18}$ to pass data between the top and middle levels.

In this study, block fuel is minimized by varying the structural weight, $W_{s}$, and the thickness to chord ratio, $t / c$, of the wing. The cruise Mach number is set to an artificially high value to test the methods ability, using $t / c$ as a design variable, to trade structural weight reduction for wave drag increase.

Constraints on the optimum solution include lower limits on range, missed approach climb gradient thrust, and second segment climb gradient thrust, and upper limits on landing approach speed and takeoff and landing field length. One additional constraint is computed from data returned by the lower levels. This constraint is a first order Taylor series approximation representing the structural load carrying capacity of the wing as determined by detailed analyses of the wing skin stiffened cover panels. The linearized expression is

$$
g_{\Omega}=\Omega+\frac{d \Omega}{d W_{s}} \Delta W_{s}+\frac{d \Omega}{d t / c} \Delta t / c
$$

where $\Omega$ is the optimum structural load carrying quantity at the specified initial values of $W_{s}$ and $t / c$, and $d \Omega / d W_{s}$ and $d \Omega / d(t / c)$ are optimum sensitivity derivatives computed from the lower levels. 


\section{DESCRIPTION OF LEVEL II}

The middle level of the multilevel system uses the Engineering Analysis Language (EAL) ${ }^{13}$ computer program to perform a finite element structural analysis of the wing box. EAL is also used to optimize the wing skin distribution to minimize local skin panel failure criteria. The EAL system consists of multiple processors which are invoked through user supplied commands, and a named data set library structure for interprocessor communications. An additional attribute of the system is the ability to link user supplied processors with it such that the data library may be accessed and the EAL command structure used. This permits external analysis programs to be included in EAL, such as numerical optimization codes. One such code, the Constrained Function Minimization (CONMIN) ${ }^{19}$ computer program, was installed into EAL for use by the middle level. Additional programs were included to perform data transfer between the EAL data set libraries and RIM for data transfer with the top level. EAL commands to perform the middle level finite element structural analysis, the approximate analysis based on results obtained from the bottom level, and the optimization of the wing skin distribution are stored in EAL runstream data sets. These runstreams contain all of the processor execution and data manipulation commands necessary to carry out each task, and include looping and branching capabilities to automate the analysis and optimzation processes.

The thickness distribution of the wing skins covering the structural box on the upper and lower surfaces is optimized at this level. The skins are sized to match the wing skin weight specified by the top level while maximizing the load carrying capacity of the individual stiffened cover panels. The wing skin weight is assumed to be

$$
W_{\text {skin }}=f \cdot W_{s}
$$


which is an estimation of the percentage of wing skin weight included in the total wing structural weight. The wing skin distribution is defined using two second order polynomials, one for the outboard and one for the inboard region of the wing. The division between inboard and outboard regions occurs near the engine attach point as shown in figure 2. Skin material distribution was not allowed to vary in the chordwise direction. The constant term of the polynomial, $\mathrm{C}_{0}$, is used over the entire wing span, with independent linear and quadratic terms in each wing region. The resulting skin distribution equations are:

$$
\begin{aligned}
& t_{\text {outboard }}=C_{0}+C_{1}(1-\beta)+C_{2}(1-\beta)^{2} \\
& t_{\text {inboard }}=C_{0}+C_{3}(1-\beta)+C_{4}(1-\beta)^{2}
\end{aligned}
$$

The $t_{\text {inboard }}$ equation corresponds to a range of $\beta$ from 0 to .4333 and the $t_{\text {outboard }}$ equation for $\beta$ from .4333 to 1.0. From equation (3), the skin panel thickness can be computed for any panel on the wing. The five coefficients $\mathrm{C}_{0}$ through $\mathrm{C}_{4}$ are used as design variables during the optimization. The function to be minimized is a cumulative constraint which measures the load carrying capacity of each of the stiffened wing panels. The function is

$$
\Omega=1 / p \log _{\theta}\left(\sum e^{p \omega_{i}}\right)
$$

which is a Kreisselmeier-Steinhauser function(K-S) ${ }^{5}$ of the bottom level objective functions. The derivatives of $\Omega$ with respect to the coefficients $C_{0}-C_{4}$ are computed by differentiating equation (4), where $d \omega_{i} / \mathrm{dC}_{l}$ are the sensitivity derivatives computed at the bottom level. 
The only constraints used at the middle level are equality constraints on the total wing structural weight $W_{s}$ and the wing thickness to chord ratio $t / c$. Since these are equality constraints, they must be handled differently from the inequality constraint formulation that program CONMIN is designed to use. The $t / c$ equality constraint is easy to satisfy because the middle level design variables are not functions of the wing depth. Therefore, when the middle level analysis is entered, the finite element model of the wing is modified to have the specified $t / c$. The wing structural weight, on the other hand, is a function of the middle level design variables. The equality constraint on $W_{s}$ is satisfied by making one of the design variables a dependent variable. The coefficient $C_{0}$ was selected to be the dependent design variable because it affects the entire wing. The wing skin weight is defined by the expression

$$
W_{\text {skin }}-\{t\}^{\top}\{A\} \rho_{d}
$$

Substituting equation (3) into (5) and performing the vector multiplications simplifies the expression to

$$
W_{\text {skin }}=C_{0} A_{0}+C_{1} A_{1}+C_{2} A_{2}+C_{3} A_{3}+C_{4} A_{4}
$$

By substituting equation (2) into (6) and rearranging the resulting expression, the equality constraint for $W_{s}$ will be satisfied by

$$
C_{0}=\frac{\left(f \cdot W_{s}-C_{1} A_{1}-C_{2} A_{2}-C_{3} A_{3}-C_{4} A_{4}\right.}{A_{0}}
$$


This reduces the number of independent design variables at the middle level to four, the coefficients $C_{1}$ through $C_{4}$. No constraints were imposed on the non-negativity of the skin thicknesses, if a negative value of thickness should occur the system would terminate execution and report the error. Since there are no other constraints or move limits, the optimization problem reduces to an unconstrained problem. This fact simplifies the minimization process and has a large impact on the computation of sensitivity derivatives at the middle level.

Sensitivity derivatives are computed for the optimum objective function with respect to the top level design variables $W_{s}$ and $t / c$ for use at the top level. Since the middle level optimization problem is unconstrained, the sensitivity derivatives are determined by simply differentiating the objective function with respect to $W_{s}$ and $t / c$. The derivative of the objective function with respect to $t / c$ is computed by finite differences. A new finite element model is generated by perturbing $t / c$. A static solution is obtained for the perturbed model including the new stress state. The change in $t / c$ only affects stress and to a lesser degree, the wing panel dimensions. We neglect the panel dimension changes as second order effects, therefore the sensitivity derivative reduces to

$$
\begin{aligned}
& \frac{d \Omega}{d t / c}=D K S\left(\omega_{i}, \frac{d \omega_{i}}{d t / c}\right) \\
& \frac{d \omega_{i}}{d t / c}=\Sigma \frac{d \omega_{i}}{d \sigma_{m}} \cdot \frac{d \sigma_{m}}{d t / c}
\end{aligned}
$$

where $d \omega_{j} / d \sigma_{m}$ are the sensitivity derivatives with respect to stress as computed at the bottom level for each panel, and $d \sigma_{m} / d(t / c)$ are the finite difference derivatives 
computed by perturbing the finite element model at the middle level. The sensitivity derivative of the objective function with respect to $W_{s}$ is computed analytically by using the relationship between the design variables and $W_{\mathrm{s}}$.

$$
\begin{gathered}
\frac{d \Omega}{d W_{s}}=D K S\left(\omega_{i}, \frac{d \omega_{i}}{d W_{s}}\right) \\
\frac{d \omega_{i}}{d W_{s}}=\frac{d \omega_{i}}{d C_{0}} \cdot \frac{d C_{0}}{d W_{s}}
\end{gathered}
$$

where $d \omega_{i} / d C_{0}$ are sensitivity derivatives returned from the bottom level and $d C_{0} / d W_{s}$ is computed by differentiating equation (7). Only the dependent coefficient $C_{0}$ appears in this equation since $\mathrm{C}_{1}$ through $\mathrm{C}_{4}$ are independent of $\mathrm{W}_{s}$. The resulting two senstivity derivatives $d \Omega / d W_{s}$ and $d \Omega / d(t / c)$ and the optimum objective function $\Omega$ are passed up to the top level for inclusion in that level's optimization. These three quantitites approximate the behavior of the lower level in a piecewise linear fashion, and, in general, care must be taken to ensure that sufficiently small changes of $W_{s}$ and $t / c$ are used at the top level to protect the validity of the approximation. For the problem used in this report, the system showed sufficient robustness to eliminate the need for move limits.

\section{DESCRIPTION OF LEVEL III}

The bottom level of the three level system uses an extended version of the OPCOM ${ }^{14}$ computer program to perform a detailed stress and buckling analysis on a rectangular panel stiffened with equally spaced $Z$ or $J$ type stiffeners. Both the skin and the stiffener must use a metal material. Figure 3 shows a panel with the stiffener 
details. Extensions to OPCOM allow for its inclusion into EAL as an external processor. This extension facilitates data transfer between levels 2 and 3 utilizing the EAL data set library system. Additional extensions were included for sensitivity derivatives of the optimum objective function with respect to middle level parameters, and to better handle multiple loading conditions.

The optimization problem to be solved is a minimization of a K-S function of 13 behavior response quantities for a given set of loads and other parameters. The design variables at the bottom level are the 3 stiffener thicknesses and the three stiffener widths, as seen in figure 3. Stiffener spacing, panel dimensions, panel thickness, and applied loads are held constant. The objective function is formulated as a single K-S function which contains a set of 13 behavior response equations for every load case. The $\mathrm{K}-\mathrm{S}$ equation is

$$
\omega_{i}=1 / \rho \log _{e}\left(\sum e^{\rho g} g_{j k}\right)
$$

where $g_{j k}$ is the $j^{\text {th }}$ behavior response equation evaluated for the $k^{\text {th }}$ applied load case. This single response equation, when minimized, produces a stiffened panel design which has the smallest violation of the individual behavior responses for the given set of parameters and loads. A positive value for $\omega_{i}$ indicates panel failure, and a negative value indicates an overdesigned panel.

Constraints at this level include both equality and inequality types. There are three inequality constraints which ensure that thickness to width ratios for the two stiffener flanges and the stiffener web stay within acceptable limits. Minimum gages are also imposed on all design variables. Since the middle level design variables are fixed parameters on the bottom level, equality constraints are used to satisfy this 
requirement. The coefficients $\mathrm{C}_{0}$ through $\mathrm{C}_{5}$ from the middle level determine a fixed skin thickness. The equivalent stiffener thickness is assumed to be 60 percent of the skin thickness. Applied in-plane panel loads from the middle level are computed from the static solution at the middle level, and are also held constant at the bottom level. The skin thickness and applied loads are held constant by directly specifying them. The smeared stiffener thickness is converted to a single stiffener cross sectional area, and this area is then used to eliminate one bottom level design variable. The web thickness was chosen to be the dependent variable, and the equality satisfied by the following equation:

$$
t_{\text {web }}=\frac{A_{\text {stif }}-A_{\text {flanges }}}{h_{\text {web }}}
$$

This leaves five independent design variables to be used in the minimization process. Following a successful optimization, sensitivity derivatives are computed so that the behavior of the optimum solution at the bottom level can be approximated.

Sensitivity derivatives of the optimum solution are required to formulate the approximate piecewise linear analysis problem at the middle level. In this case, a sensitivity of optimum derivation is necessary due to the inequaltiy constraints involved. To obtain the sensitivities, the computer program SENSUB ${ }^{3}$ is used. Derivatives of the objective function $\omega_{i}$ are obtained with respect to the skin thickness, smeared stiffener thickness, and each applied force. The following equations describe the relationship between these derivatives and those required by the middle level.

$$
\frac{d \omega_{i}}{d C_{\ell}}=\frac{d \omega_{i}}{d t_{\text {skin }}} \cdot \frac{d t_{\text {skin }}}{d C_{\ell}}+\frac{d \omega_{i}}{d t_{\text {stif }}} \cdot \frac{d t_{\text {stif }}}{d C_{\ell}}+\sum \frac{d \omega_{i}}{d \sigma_{m}} \cdot \frac{d \sigma_{m}}{d C_{\ell}}
$$




\section{FINITE ELEMENT MODEL}

The finite element representation of the $L-1011-500$ transport aircraft was developed by Lockheed-California Company for analysis by the NASTRAN 20 program used in the PADS ${ }^{6}$ system. Since the focus is on wing design, a fairly detailed model is used for the wing structure and the regions of the fuselage necessary to get proper representation of the wing-body intersection. The wing and wing-body intersection are modelled primarily with rod and membrane panel elements. The remainder of the structure (fore and aft fuselage, empennage, engine, and landing gear) is modelled using beam elements. This NASTRAN model was converted to an EAL compatible model at the NASA/LaRC for use in the work reported herein. A total of 641 joints, 170 beam elements, 775 rod elements, and 786 membrane elements are used for the symmetric half model. During design studies, only the cover panels in the upper and lower surfaces of the main wing box (216 elements) were resized (figure 4).

Static loads applied to the finite element model were provided under contract NAS1-16794 in the form of constant load vectors. The load cases used to test this system correspond to trimmed $2.5 \mathrm{~g}$ symmetric maneuver conditions. Loads were not modified during the design process.

\section{RESULTS AND DISCUSSION}

The multilevel procedure discussed in this report has been applied to the wing sizing of an L-1011-500 transport aircraft configuration. Four cases are presented, each differing in the starting points of top and middle level design variables. Table 1 
shows the configuration data for the L-1011-500 version used in this study. Table 2 shows the initial and final values of the required block fuel consumption, wing weight, thickness to chord ratio, and the number of complete three level cycles required for convergence for each of the 4 cases shown. Cases 1 through 3 all use a realistic initial wing skin thickness distribution as the middle level starting point. Case 4 uses an unrealistic skin distribution so that the robustness of the middle level optimization scheme is demonstrated. All four cases converged to approximately the same final values of wing weight, thickness to chord ratio, and block fuel. Figure 5 shows the initial and final wing skin thickness distributions for case 3 . This case has a realistic initial skin distribution and upon convergence has a similar distribution optimized to the correct weight. In figure 6 , the initial and final skin distributions for case 4 are shown. This case has an initial skin distribution which is very unrealistic, yet the middle level optimization procedure demonstrates sufficient robustness to achieve approximately the same final skin distribution as that of figure 5 . Figures 7 and 8 show the convergence history of the top level objective function, which is the block fuel required to perform the mission. Figures 9 through 12 show the convergence history for the top level design variables, figures 9 and 10 for the wing structural weight and figures 11 and 12 for the thickness to chord ratio. Each of the four cases was optimized for 11 to 14 cycles to test the stability convergence. In all cases, 9 to 14 cycles produced a design which no longer changed any of the design variables. Realistic convergence criteria would result in far fewer cycles being required. Oscillations of the top level design variables and the objective function are primarily due to the lack of move limits on the design variables. Move limits were not used in order to reduce the tendency of some cases to converge to local minima at the cost of inducing oscillations in other cases. The quality of the sensitivity derivatives computed at the lower levels was satisfactory to permit this. 
Computer resources required for the multilevel system are shown in table 3 , broken down by level and by CPU time and mass storage activity. All data are for a Cyber 175 mainframe computer under the Network Operating System (NOS) ${ }^{21}$ version 2.4 located at the NASA/LaRC. The top level utilizes 61 percent of the total Central Processing Unit (CPU) time, and has very little mass storage activity. The middle level typically utilizes 36 percent of the total CPU time and 99 percent of the mass storage activity, with the majority of both used for derivative calculations. The optimization coupled with the linearized analysis is quite efficient, utilizing only 2 percent of the middle level's CPU time. The bottom level uses the other 3 percent of total CPU time and a negligible amount of mass storage activity, even though 216 individual wing skin panel optimizations are performed.

\section{CONCLUSIONS}

A multilevel procedure has been applied for the sizing of a large transport aircraft wing for both mission performance and structures criteria. The three level system developed performs a mission analysis at the top level, a finite element analysis at the middle level, and a stiffened panel analysis at the bottom level. Each level performs an optimization to size a set of local design variables using nonlinear programming techniques. Four selected starting conditions were presented to show system performance. In each case, the optimum block fuel consumption, wing structural weight, wing thickness to chord ratio, and wing skin thickness distribution converged to similar designs. The method made it possible to use very large numbers of design variables (1303) and constraints (1950). It also demonstrated that a mathematical link can be established between a design detail, such as structural

cross-sectional sizing, and the aircraft performance, such as the fuel consumption. 


\section{REFERENCES}

1. Haftka, Raphael T., Kamat, Manohar P.: Elements of Structural Optimization, Martinus Nijhoff Publishers, 1985.

2. Vanderplaats, Garret N.: Numerical Optimization Techniques for Engineering Design with Applications, McGraw-Hill Book Company, 1984, pp. 217-225.

3. Sobieszczanski-Sobieski, J., Barthelemy, J.-F., Riley, K.M.: "Sensitivity of Optimum Solutions to Problem Parameters", AIAA Journal, Vol. 20, September 1982, pp. 1291.

4. Frank, P.: Introduction to System Sensitivity Theory, Academic Press, 1978, pg. 279.

5. Kreisselmeier, G., Steinhauser, R.: "Systematic Control Design by Optimizing a Vector Performance Index", IFAC Symposium on Computer Aided Design of Control Systems, Zurich, Switzerland, Aug. 29-31, 1979.

6. Radovcich, N.A.: "Preliminary Aeroelastic Design of Structures [PADS] Methods Development and Application". Aeroelastic Considerations in the Preliminary Design of Aircraft, AGARD-CP-354, Sept. 1983, pp. 1-1 - 1-29. 
7. Radovcich, Nick A.: "Some Experiences in Aircraft Aeroelastic Design Using Preliminary Aeroelastic Design of Structures [PADS]". Recent Experiences in Multidisciplinary Analysis and Optimization, Jaroslaw Sobieski, compiler, NASA CP-2327, Part 1, April 24-26, 1984, pp. 455-503.

8. Sobieszczanski-Sobieski, J.: "A Linear Decomposition Method for Large Optimization Problems--Blueprint for Development", NASA TM-83248, February 1982.

9. Sobieszczanski-Sobieski, J., Barthelemy, J.-F.: "Improving Engineering System Design by Formal Decomposition, Sensitivity analysis, and Optimization", Proceeding of the International Conference of Engineering Design, Hamburg, W. Germany, Aug. 26-28, 1985.

10. Sobieszczanski-Sobieski, Jaroslaw, Barthelemy, Jean-Francois M., Giles, Gary L.: "Aerospace Engineering Design by Systematic Decomposition and Multilevel Optimization", NASA TM-85823, Jun, 1984.

11. Sobieszczanski-Sobieski, J., James, B.B., Dovi, A.R.: "Structural Optimization by Multilevel Decomposition", AIAA Journal, Vol. 23, November 1985, pp. 17751782.

12. Sobieszczanski-Sobieski, J., James, B.B., Riley, M.F.: Structural Optimization by Generalized, Multilevel Decomposition", Proceedings of the AIAAASME/ASCE/AHS 26th Structures, SDM Conference, April 1985. 
13. Whetstone, W.D.: EISI-EAL Engineering Analysis Language Reference Manual EISI-EAL System Level 2091, Engineering Information Systems, Inc., July 1983.

14. Sobieszczanski-Sobieski, Jaroslaw: "An Integrated Computer Procedure for Sizing Composite Airframe Structures". NASA TP-1300, February 1979.

15. McCullers, L.A.: "FLOPS - Flight Optimization System", CP-2327, Part 1, April 2426, 1984, pp. 395-412.

16. Feagin, Richard C. and Morrison, William D., Jr.: "Delta Method, An Empirical Drag Buildup Technique". NASA CR-151971, December 1978.

17. Fox, Richard, L.: Optimization Methods for Engineering Design, pp.104-109, Addison-Wesley Publishing Co., Reading, MA, 1971.

18. User Guide: "Relational Information Management (RIM)", Report DG-IPAD$70023 \mathrm{M}$, Sept. 1982.

19. Vanderplaats, Garret N.: "CONMIN - A FORTRAN Program for Constrained Function Minimization - Users Manual", NASA TM-X-62282, 1973.

20. The NASTRAN User's Manual, NASA SP-222(06),September 1983.

21. NOS Ver. 2 Reference Set, Control Data Corp., May 1986. 
TABLE 1. CONSTANT PARAMETERS FOR THE LOCKHEED L-1011-500

\begin{tabular}{|c|c|c|}
\hline Aspect Ratio & 7.63 & \\
\hline 1/4 Chord Sweep Angle & 35 & Degrees \\
\hline Mach Number & 0.90 & \\
\hline Total Payload & 86000 & Lbs. \\
\hline Design Range * & 4780 & N.M. \\
\hline Maximum Landing Velocity * & 151 & Kts. \\
\hline Maximum Takeoff Distance * & 9000 & Feet \\
\hline Maximum Landing Distance * & 9000 & Feet \\
\hline Missed Approach Climb Thrust * & 25000 & Lbs./Engine \\
\hline Second Segment Climb Thrust * & 25000 & Lbs./Engine \\
\hline
\end{tabular}

* Optimization Constraint Limits

TABLE 2. INITIAL AND FINAL OPTIMIZATION PARAMETERS

\begin{tabular}{|c|c|c|c|c|c|c|c|}
\hline \multirow[b]{2}{*}{$\begin{array}{l}\text { Case } \\
\text { Number }\end{array}$} & \multicolumn{3}{|c|}{ INITIAL VALUES } & \multicolumn{3}{|c|}{ FINAL VALUES } & \multirow[b]{2}{*}{$\begin{array}{l}\text { Cycles to } \\
\text { Converge }\end{array}$} \\
\hline & $\begin{array}{c}\text { Block } \\
\text { Fuel } \\
\text { (LBS) }\end{array}$ & $t / c$ & $\begin{array}{l}\text { Wing } \\
\text { Weight } \\
\text { (LBS) }\end{array}$ & $\begin{array}{c}\text { Block } \\
\text { Fuel } \\
\text { (LBS) }\end{array}$ & $t / c$ & $\begin{array}{l}\text { Wing } \\
\text { Weight } \\
\text { (LBS) }\end{array}$ & \\
\hline 1 & 171677 & 0.0800 & 40000 & 182974 & 0.0923 & 53099 & 14 \\
\hline 2 & 209901 & 0.1100 & 65000 & 183144 & 0.0924 & 53311 & 9 \\
\hline 3 & 183235 & 0.0800 & 70000 & 183025 & 0.0924 & 53051 & 10 \\
\hline 4 & 183235 & 0.0800 & 70000 & 183128 & 0.0926 & 52948 & 12 \\
\hline
\end{tabular}


TABLE 3. COMPUTER RESOURCE CHART FOR A TYPICAL 3 LEVEL CYCLE

\begin{tabular}{|c|c|c|c|}
\hline & CPU SECONDS & LO COUNTS & \\
\hline LEVEL 1 & & 480 & --- \\
\hline \multirow[t]{6}{*}{ LEVEL 2} & Model Setup & 12 & 750 \\
\hline & Static Analysis & 36 & 2231 \\
\hline & Stress Derivatives & 180 & 11254 \\
\hline & Optimization (8 iterations) & 6 & 2996 \\
\hline & Sensitivity Derivatives & 49 & 3271 \\
\hline & TOTAL & 283 & 20502 \\
\hline LEVEL 3 & 216 Panels & 20 & 69 \\
\hline \multicolumn{2}{|c|}{ TOTAL FOR THREE LEVELS } & 783 & 20571 \\
\hline
\end{tabular}




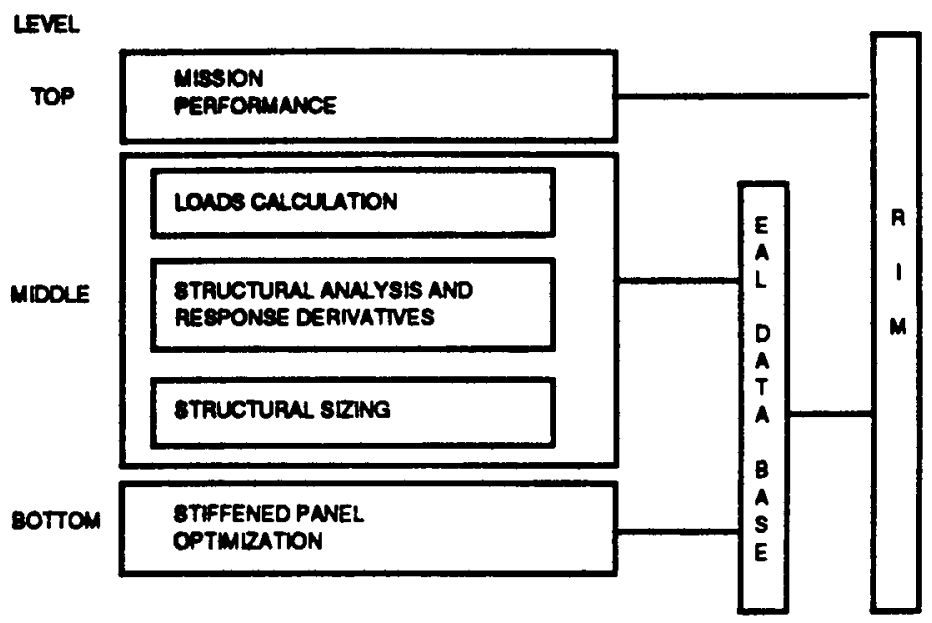

Figure 1. Block Diagram of Multilevel Scheme

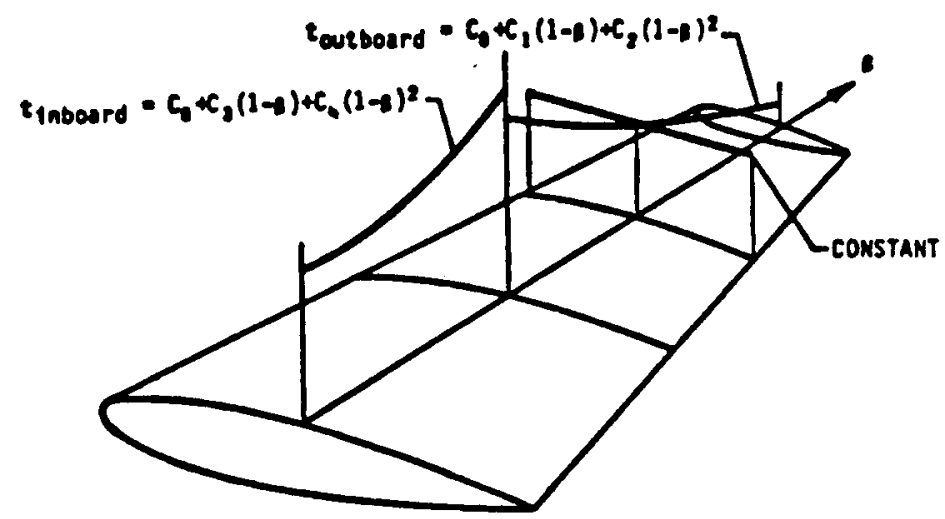

Figure 2. Wing Skin Distribution
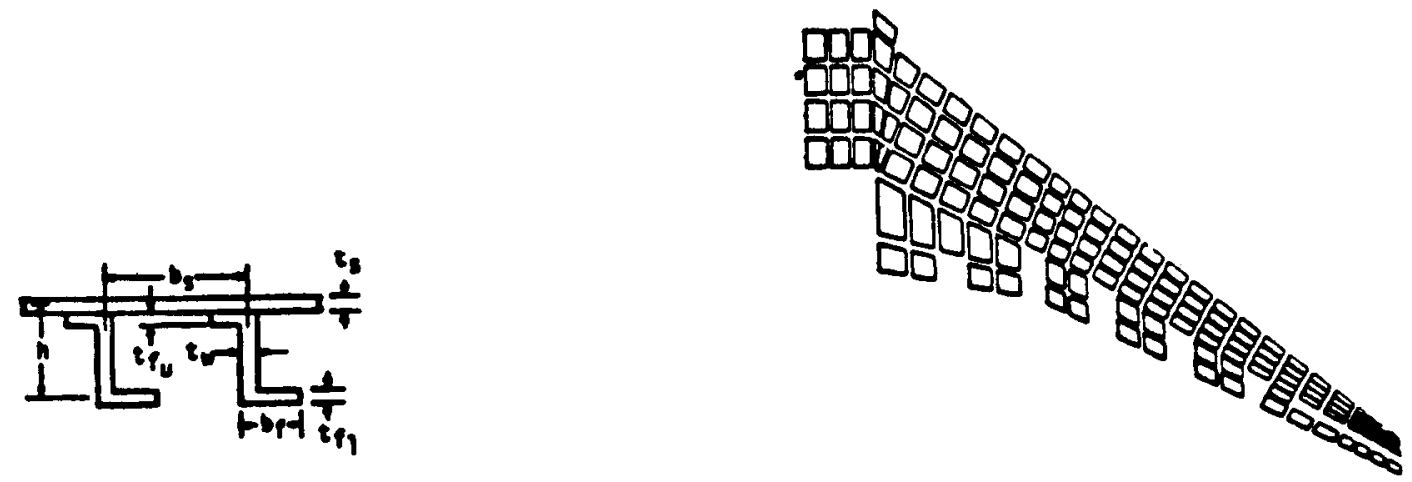

Figure 3. Stiffened Panel Design Variables

Figure 4. Wing Cover Panels 


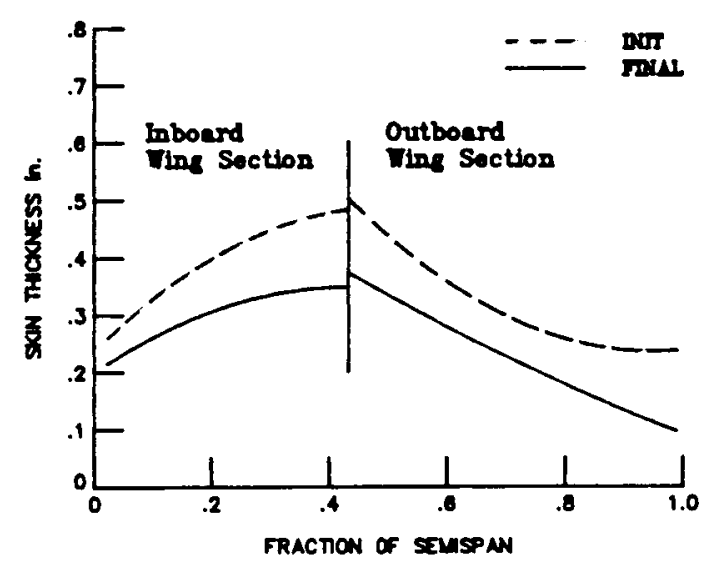

Figure 5. Skin Thickness Distribution, With Realistic Initial Conditons

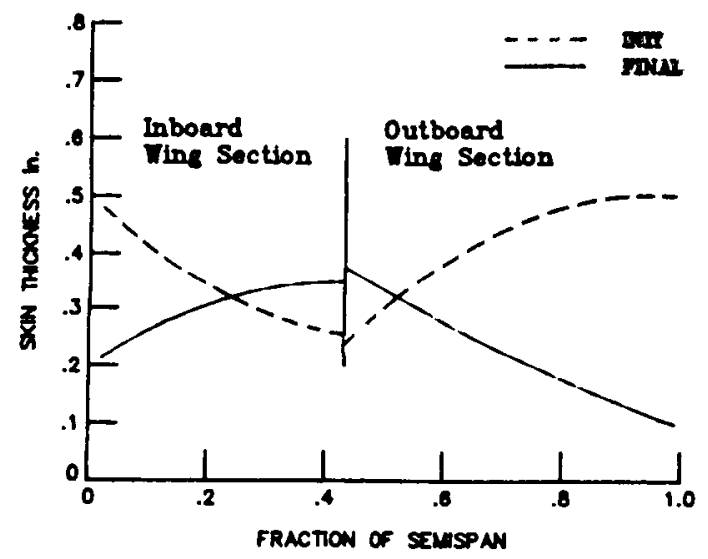

Figure 6. Skin Thickness Distribution, With Unrealistic Initial Conditions

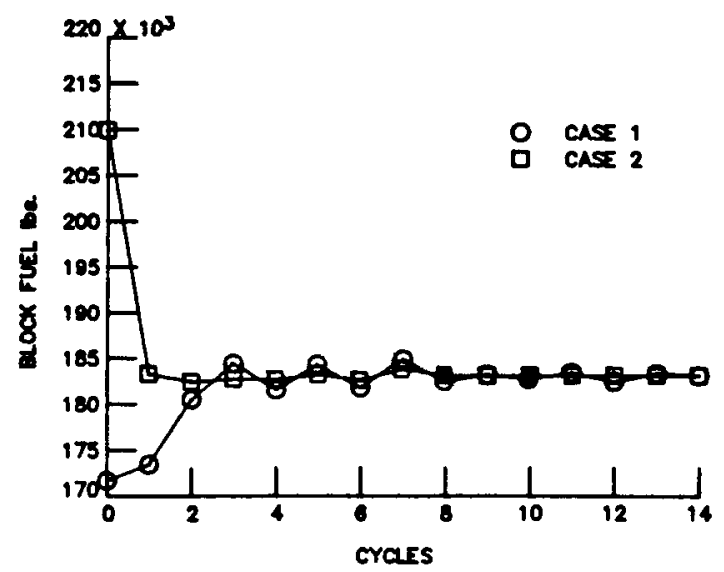

Figure 7. Block Fuel Convergence

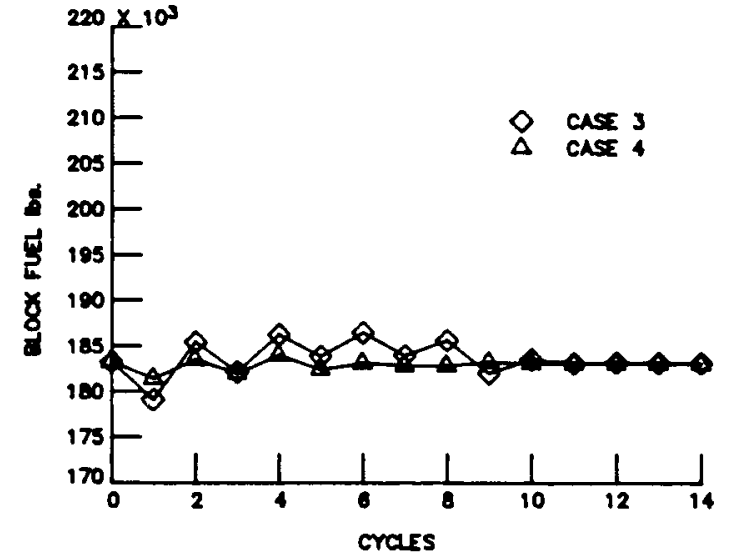

Figure 8. Block Fuel Convergence

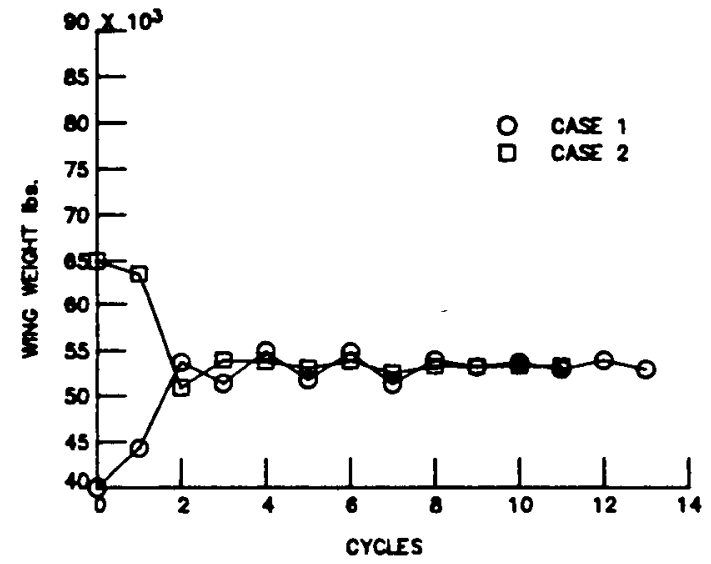

Figure 9. Wing Weight Convergence

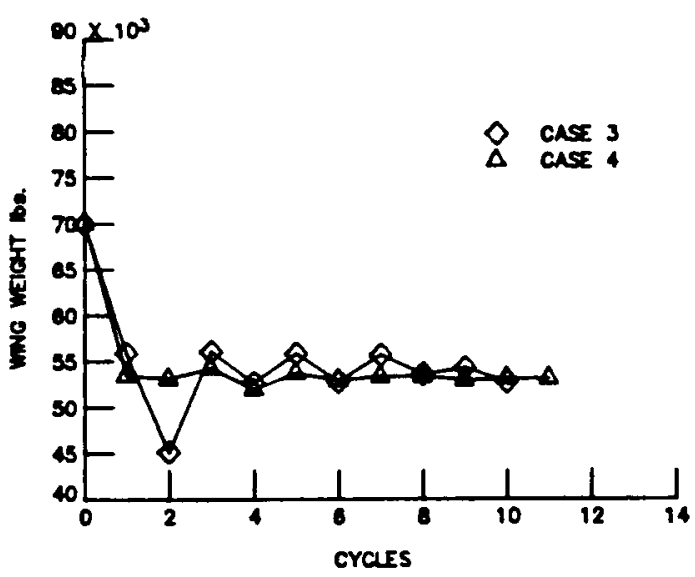

Figure 10. Wing Weight Convergence 


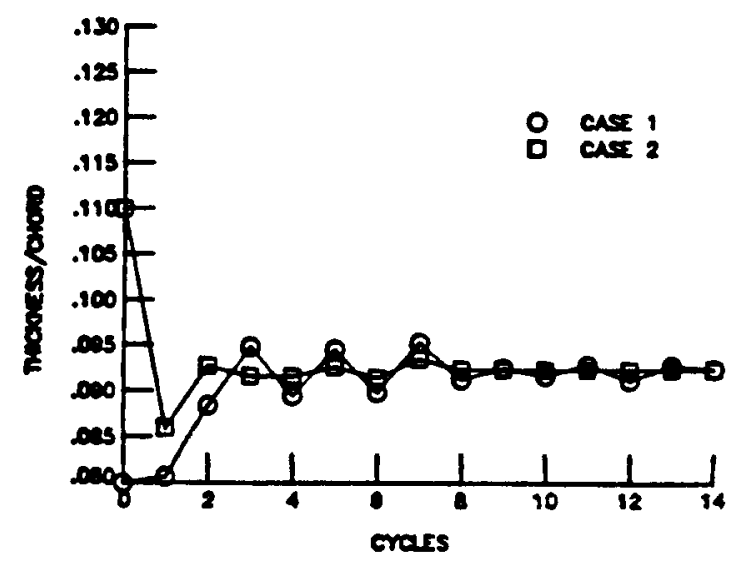

Figure 11. Thickness/Chord Convergence

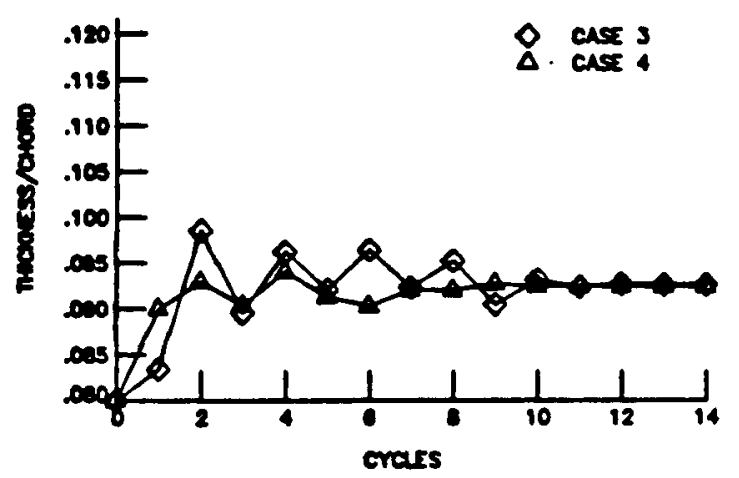

Figure 12. Thickness/Chord Convergence 


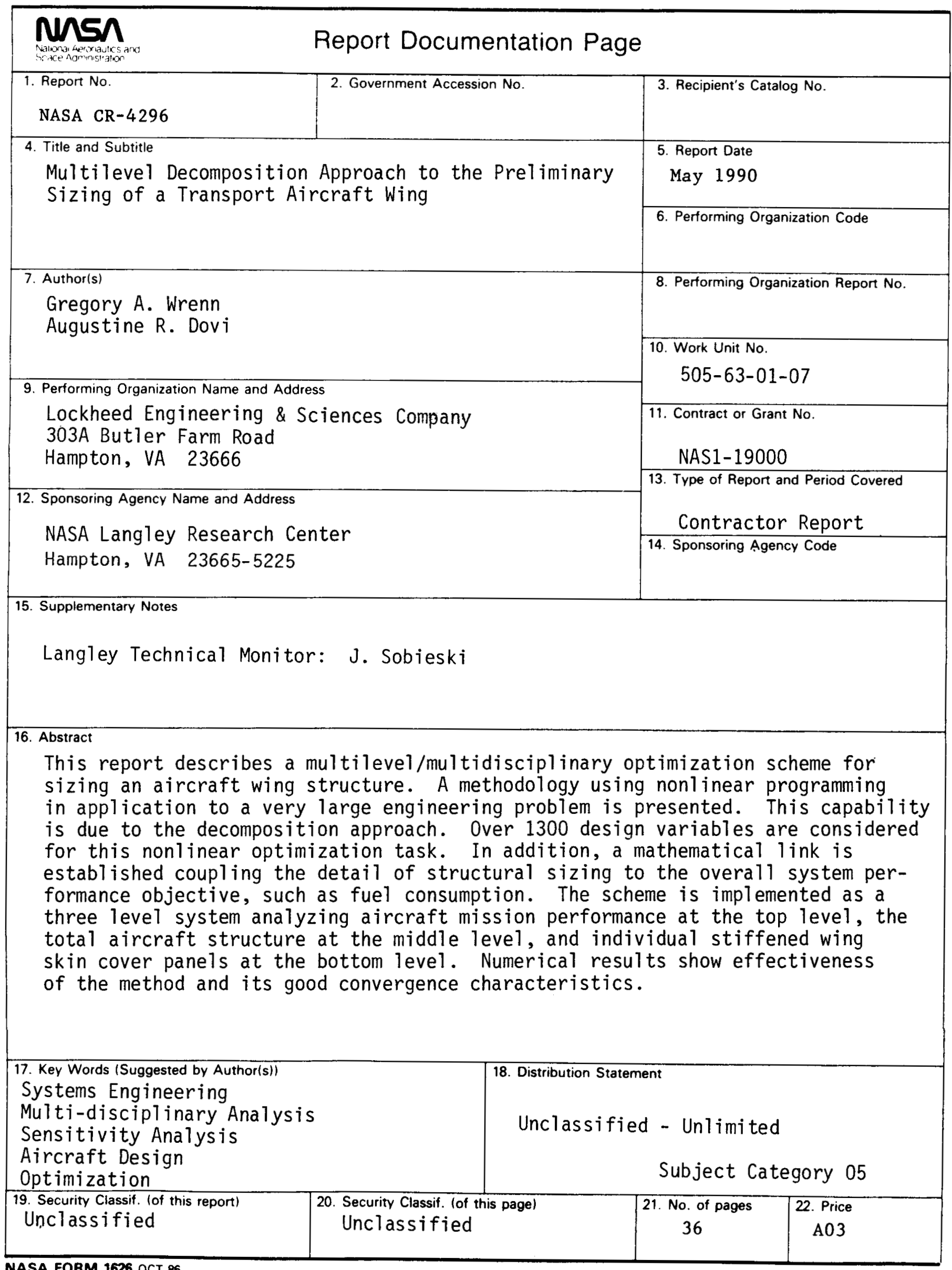

